\title{
Motivando la construcción de conocimiento colectivo a través del juego: un análisis de caso implementando medios físicos
}

\author{
Maria Julieta Lombardelli ${ }^{(1)}$, Diego Torres ${ }^{(2)}$
}

y Alejandro Fernandez ${ }^{(3)}$

\begin{abstract}
Resumen: Actualmente, el juego es analizado como un elemento importante para motivar la interacción de usuarios en diferentes tipos de entornos. Este análisis ha derivado en promover diferentes estrategias para adaptar elementos propios de los juegos y los videojuegos en contextos que no están diseñados como tal, pero en los que se busca estimular la participación de los usuarios. Una estrategia al aplicar elementos de juego es considerar un modelo centrado en la experiencia del usuario.

Sin embargo, para establecer un diseño de juego en contextos en donde prevalece el aspecto colaborativo, se requiere abordar estrategias específicas centradas en su relación con la comunidad a la que pertenece. Este artículo presenta el diseño de un entorno ludificado basado en el marco conceptual G.A.M.E. acrónimo de recopilación, análisis, modelado y ejecución (gathering, analysis, modeling and execution), aplicado a una comunidad de construcción colaborativa de conocimiento sobre Buenas Prácticas Hortícolas, complementado, además, con una interfaz física.
\end{abstract}

Palabras clave: ludificación - ciencia ciudadana - trabajo colaborativo - Wikipedia; marco conceptual

[Resúmenes en inglés y portugués en la página 91]

(1) Lic. En Diseño Multimedial, Doctoranda en Artes, Docente y artista multimedia. Trabaja en las intersecciones del arte, la ciencia y la tecnología. Actualmente investiga la relación entre Ludificación y Ciencia Ciudadana. Coordinadora del nodo de Innovación tecnológica en NEA y CSLP.

(2) Doctor en Ciencias Informáticas. En 2014 obtuvo los títulos de Doctor en Ciencias Informáticas por la Universidad Nacional de La Plata y Doctor de la Universidad de Nantes. Sus temas de investigación se encuadran dentro de Citizen Science, Open Science, Social Semantic Web, Semantic Web, Linked Open Data y sistemas de recomendación.

${ }^{(3)}$ Doctor en Ciencias Informáticas. Profesor Adjunto con Dedicación Exclusiva en la Facultad de Informática de la Universidad Nacional de La Plata (UNLP). Se graduó en 1999 
como Licenciado en Informática en la UNLP. En 2005 obtuvo el título de Doctor en la FernUnivesität Hagen, en Alemania, trabajando en varios temas relacionado al CSCW y la Ingeniería de Software.

\section{Introducción}

La ludificación es una herramienta que se implementa actualmente en los más diversos ámbitos. Como concepto refiere a la implementación de elementos que son tradicionales de los juegos en espacios o entornos que no fueron diseñados originalmente con ese fin. El objetivo principal por el cual se aplica esta estrategia es motivar a los usuarios a que cumplan un objetivo tal como realizar alguna tarea, permanecer más tiempo en algún entorno, participar más activamente de una tarea, etc. (Deterding et al., 2011).

Por lo tanto, al ser un recurso adaptable a circunstancias completamente diferentes entre sí, surgen numerosos estudios para analizar cuáles son los principales elementos que formalizan la ludificación como disciplina. Entre estos análisis cabe mencionar la propuesta que profundiza en conceptualizar una ciencia de la ludificación, que se diferencie de una ciencia del juego (Landers et al., 2018).

De esta forma, la ludificación, con sus características formales y sus particularidades adaptativas, se convierte en una potente herramienta motivacional pero que requiere de un análisis de sus alcances y sus límites para poder ser implementada adecuadamente. Como una arquitectura persuasiva, el éxito en el diseño de la misma está determinado por el alcance del conocimiento sobre las características particulares de las circunstancias en donde es implementada (Cugelman, 2013).

Como parte del proceso de diseño de una ludificación para un contexto determinado, la selección de una marco o estructura de trabajo (framework en inglés), es determinante para establecer cuáles de los variados elementos que forman parte de la ludificación son los adecuados según cada caso. En cada estructura de trabajo, los elementos clásicos que constituyen a la ludificación se relacionan en forma diferenciada. (Azouz \& Lefdaoui, 2018).

Una de las áreas en donde se implementan estrategias de ludificación, presentando un crecimiento sostenido en los últimos años, es en entornos de trabajo colaborativo, entre los que se encuentran los denominados crowdsourcing (Morschheuser et al., 2016). Estos espacios se caracterizan por la participación de un grupo de personas o colectivo de personas que se reúne para llevar a cabo una tarea en común, trabajando en forma abierta y colaborativa. (Crowdsourcing, 2020). Entre estos espacios se distinguen la recolección y procesamiento de datos como en la Ciencia Ciudadana u otros espacios de creación de conocimiento basados en tecnologías digitales Web, por ejemplo, Wikipedia.

En este artículo se presenta un diseño de ludificación para una comunidad de conocimiento que se desarrolla en una wiki colaborativa. Este diseño se analiza desde una estructura de trabajo que se presenta específica para implementar en entornos y comunidades colaborativas llamada G.A.M.E. (gathering, analysis, modeling and execution) (Brito et al., 2015). 


\section{El juego en entornos colaborativos: antecedentes en Wikipedia}

Las comunidades de creación de conocimiento (Knowledge Building Community, KBC) son espacios cuyo objetivo principal es producir contenido a través de contribuciones individuales. La complejidad de la tarea de generar nuevo conocimiento disminuye dado que los resultados son la combinación de múltiples contribuciones en lugar de realizarlas sólo una persona (Torres et al., 2017).

Entre los ejemplos de KBC se encuentran las comunidades de prácticas (Community of Practice, CoPs), caracterizadas como grupos de personas que comparten prácticas en común para desarrollar su conocimiento juntos y a través de sus interacciones (Wenger, 2011). Desde el desarrollo de la web, muchas de estas comunidades se forjaron en espacios virtuales y sitios web, siendo Wikipedia la de mayor alcance a nivel mundial (Zhao \& Bishop, 2011).

Wikipedia es una enciclopedia web escrita por los usuarios de internet, en forma abierta y colaborativa. Cualquier persona que se registre en el sitio Web, puede crear, editar y comentar los artículos en cualquier idioma. Está basada en la tecnología MediaWiki, que sirve para crear sitios web colaborativos llamados wikis (Wikipedia, 2020).

Uno de los grandes desafíos que tiene Wikipedia en cuanto enciclopedia libre es asegurar la calidad de los artículos publicados. Para esto desarrolló una política de revisores que forman parte de la misma comunidad de Wikipedia para que evalúen y postulen los artículos más completos y mejor redactados categorizándolos con una insignia, en este caso una estrella, que significa que es un "Artículo destacado" (Featured article) (Featured article, 2020). Sin embargo, este criterio demanda de una continua revisión de los patrones que se utilizan para determinar la calidad en los artículos por parte de los editores (Wöhner \& Peters, 2009; Lindsey, 2010).

Desde esta arquitectura dinámica y de continuo crecimiento, Wikipedia requiere de una participación activa y comprometida por parte de sus colaboradores. En este sentido, los videojuegos se presentan como una herramienta motivacional para acercar a los usuarios pautas para la edición y participación. Tal es el caso que se ensayó con el videojuego Wikipedia Adventure, en donde el usuario puede aprender cómo se usa Wikipedia por primera vez a través de un videojuego completo, con una estética y una narrativa que se desprende de la wikipedia, y que utiliza un software como extensión, es decir, que no corre directamente sobre Wikipedia, sino que utiliza el perfil del usuario, pero a través de otro software (Narayan et al., 2017).

En la Ilustración 1 se observa parte de la interfaz de Wikipedia Adventure, en donde destaca una estética que se diferencia de la interfaz de Wikipedia, al proponer mediante la narrativa y los gráficos utilizados, su propio espacio para desarrollar la experiencia de aprendizaje. De esta manera Wikipedia Adventure es un tutorial y al mismo tiempo un videojuego completo para el aprendizaje. Además de realizar una introducción a las mecánicas de edición de Wikipedia, mientras se recorren las diferentes misiones, explica la filosofía de Wikipedia (The Wikipedia Adventure-Outreach Wiki, s. f.). 


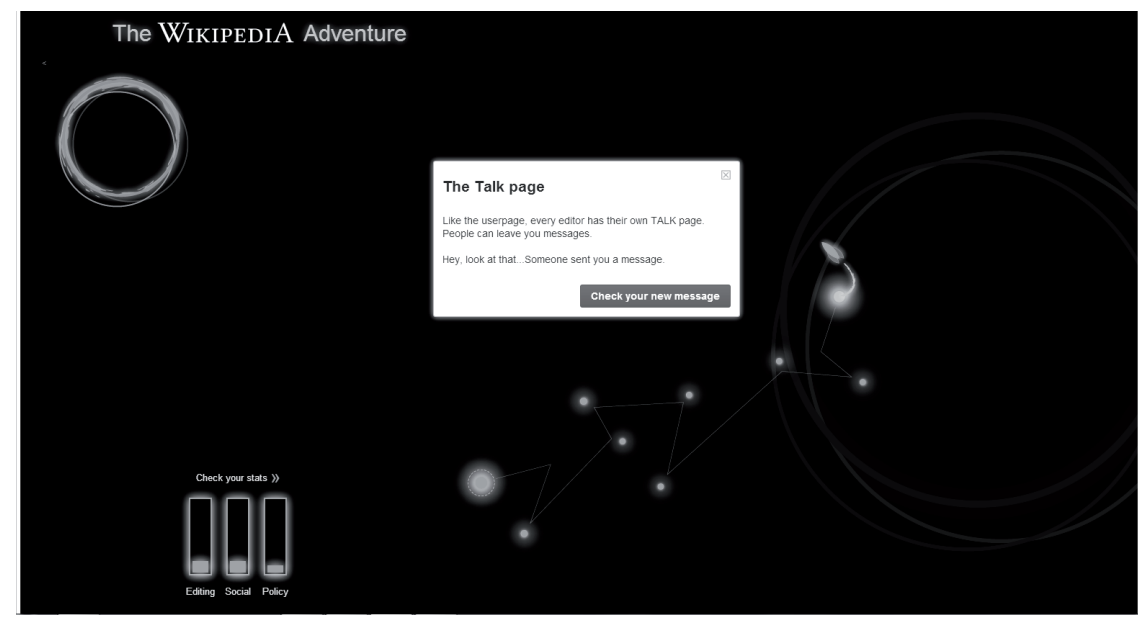

Ilustración 1. Captura de pantalla de Wikipedia, Artwork by Heather Walls - https://en.wikipedia.org/ wiki/WP:TWA/2/Start?tour=twa2\&step=2, CC BY-SA 3.0, https://commons.wikimedia.org/w/index. php?curid $=27264150$

Otro abordaje sobre Wikipedia a través de una estrategia de juego tiene relación con proyectos para ludificar escenarios específicos de la misma. Tal es el caso del estudio de ludificación realizado sobre Wikimedia España, en donde a través de dos tipos de concursos específicos se propone a los usuarios de la comunidad realizar nuevos artículos y subir más imágenes a la plataforma. El sistema para motivar fue la implementación de elementos de juego tales como puntos, tablas de posiciones y recompensas (Oceja \& Obregón Sierra, 2018).

Siguiendo esta línea en donde se aborda espacios específicos dentro de la estructura de Wikipedia, se encuentra la serie de mini juegos propuestos por Wikimedia Foundation para editar eventos puntuales como fechas o imágenes, motivando a mejorar la calidad del contenido de los artículos (Wikidata-The Distributed Game, s. f.).

Por último, Wikipedia ofrece anualmente un campeonato para editores llamado WikiCup con el objetivo de "fomentar la creación y mejora de contenido y hacer que la edición en Wikipedia sea más divertida" (WikiCup, 2020). Este campeonato tiene la cualidad de que los editores que desean competir deben registrarse en la copa al comenzar cada año y de esta manera se contabilizan las ediciones que realiza en la plataforma Wikipedia regularmente generando diferentes puntajes. Esta forma de ludificación se muestra por fuera de la interfaz general de Wikipedia, contando con un espacio específico destinado a la misma.

Cabe destacar que, en los ejemplos mencionados, se contemplan los diseños de juego basándose en la experiencia del usuario como individuo, en cómo su acción inmediata impacta en Wikipedia. Sin embargo, la cooperatividad como elemento clave para el diseño de juego es aún poco explorada, surgiendo ejemplos como el de Wikimedia España, que sin embargo sigue apuntando a la premiación individual y no a un resultado grupal. 
El diseño de juego y de ludificación adquiere características específicas que depende de los objetivos que la comunidad está buscando como conjunto. Asimismo, cada grupo de comunidades tiene diferentes estrategias de relación lo que también configura su experiencia y motivaciones (Morschheuser et al., 2017). Identificar correctamente estas características puede ser clave al momento de diseñar un juego que resulte motivante y pueda sostener una participación activa a largo plazo por parte de la comunidad.

\section{Marcos conceptuales para la ludificación cooperativa}

Para que un diseño de ludificación cumpla con los objetivos que se propone, es necesario un análisis que se adecue a cada situación y contexto específico en el cual se va a implementar. Para esto existen números marcos conceptuales, frameworks en inglés que posibilitan enmarcar el diseño siguiendo determinadas pautas o pasos.

Uno de los más conocidos e implementados es el llamado Mechanics-Dynamics-Aesthetics (MDA) (Hunicke et al., 2004). En este marco se propone dividir el diseño del juego en tres ejes:

Mecánicas: es la base del juego, lo que refiere a las reglas en general.

Dinámicas: refiere a cómo se desarrollan esas reglas a lo largo del juego.

Estéticas: se relaciona con la parte emocional del juego, lo que el jugador va a sentir, o se propone que pueda experimentar.

MDA se convierte entonces en un marco genérico que por su estructura permite abordar diferentes tipos de diseños y desde el cual se han desarrollado nuevos marcos conceptuales. En este sentido, es importante destacar que el diseño de un juego es un proceso iterativo que observa y analiza mediante diferentes etapas o pasos los elementos que componen a la arquitectura del juego, articulándolos según lo demande cada situación. Sin embargo, el diseño de un videojuego o juego es diferente al proceso de diseño de una experiencia de ludificación, puesto que los objetivos en ambos casos cambian. Mientras en los videojuegos se busca principalmente que las personas que interactúen con el mismo vivencien determinadas experiencias, en un entorno ludificado el objetivo será que el usuario realice una acción específica, generalmente se trata de objetivos relacionados con las compras, los negocios, la educación y el aprendizaje, la ciencia etc. (Mora et al., 2017).

Por lo tanto, los marcos conceptuales que se pueden utilizar en el diseño de un juego, pueden ser diferentes de los que se pueden implementar en un diseño de entorno ludificado. Asimismo, dentro de los diferentes marcos conceptuales que se proponen para el diseño específico de ludificación, existen variables acordes al entorno en donde se desarrolla cada experiencia (Mora et al., 2017) (Azouz \& Lefdaoui, 2018) (Buckley et al., 2019).

Tal es el caso de las experiencias en entornos colaborativos en donde las dinámicas de las relaciones de la comunidad son las que determinan el tipo de arquitectura que puede tener un diseño de ludificación. Por ejemplo, algunos de los recursos más ampliamente utilizados en el momento de diagramar entornos ludificados son los que se relacionan con la competitividad, como las tablas de posiciones o las insignias que jerarquizan la posición individual de cada participante. Estos elementos, sin embargo, pueden ejercer el efecto 
contrario al momento de motivar una comunidad que trabaja colaborativamente. En estos casos resulta conveniente revisar qué estrategia de ludificación incentiva y promueve más un aspecto cooperativo que guíe a los usuarios ya no a competir entre ellos, sino a trabajar colaborativamente para un objetivo en común (Morschheuser et al., 2019).

De esta forma es que surgen nuevas propuestas de marcos conceptuales que observan características específicas de la colaboración y trabajo en equipo de grandes grupos de personas, por ejemplo, vinculados a la ciencia (Uchoa et al., 2013).

\section{La propuesta de G.A.M.E.}

El marco conceptual G.A.M.E., acrónimo de recopilación, análisis, modelado y ejecución (Gathering, Analysis, Modeling and Execution), está diseñado para abordar específicamente entornos de trabajo de cooperación y colaboración entre individuos (Brito et al., 2015).

La propuesta de G.A.M.E. es generar una guía paso a paso para el diseño de entornos ludificados que se diagrama de la siguiente manera:

- Recopilación (Gathering): primero, en el diseño se debe reunir toda la información necesaria para entender el escenario en donde se va a implementar la ludificación. Sistemáticamente, se propone compilar los objetivos de la comunidad incluyendo no a los objetivos individuales de sus miembros, sino al objetivo en común que tiene cada grupo. Luego se analiza cuál es la tecnología que implementan para tal fin: sus funcionalidades; $y$ finalmente se determinan los problemas que enfrenta la comunidad en conjunto.

- Análisis (Analysis): en esta etapa se analiza cuál es el principal objetivo para implementar la estrategia de ludificación. Es decir, se observa si es necesario mejorar una fortaleza o solucionar una debilidad. En este sentido, Brito et al. (2015) explica que "se puede detectar una fortaleza a partir de las funcionalidades identificadas, mientras que una debilidad proviene de problemas recopilados" (p.447)

- Modelado (Modeling): esta etapa es para diseñar las mecánicas y dinámicas de juego basándose en las características del sistema colaborativo, reunidas en la etapa anterior de análisis. Para esto se propone implementar un Modelo de interacción de juego (Gameplay Interaction Model), que sigue el esquema de la ilustración 2, basado en la propuesta de Brito et al. (2015). El jugador realiza una acción que lo lleva a un estado que genera una respuesta que vuelve al jugador. - Ejecución (Execution): la implementación y el testeo es la etapa final. Aunque son dos pasos diferentes, se incluyen en una etapa al estar íntimamente relacionados. Por lo tanto, se considera realizar primer prototipo que luego puede ser testeado con un grupo de usuarios finales de la comunidad en cuestión. 


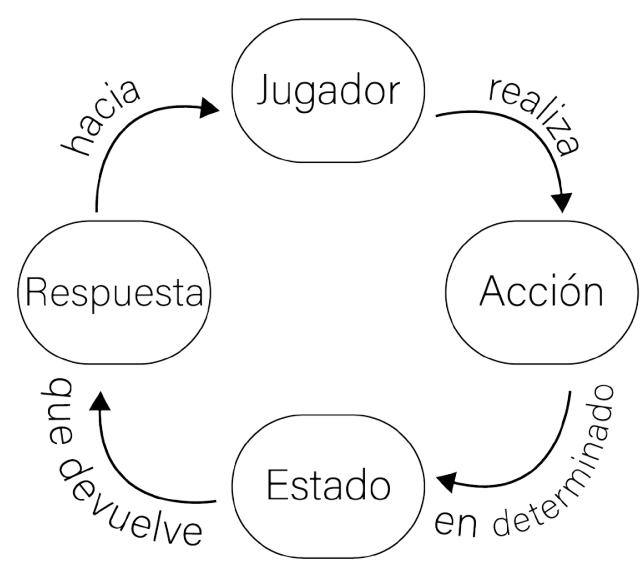

Ilustración 2: Esquema de Modelo de interacción de juego, basado en el esquema de Brito et al. (2015).

\section{Una wiki para las Buenas Prácticas Agrícolas intensivas}

Las Buenas prácticas Agrícolas (BPA) se entienden como aquellas que constituyen un corpus de acciones y conceptos para trabajar en agricultura en forma sustentable y que no resulte nociva tanto para los trabajadores y su producción (Buenas prácticas agrícolas, 2019). A partir de un proyecto de investigación y colaboración académica llamado Agroknowledge (Inteligencia Colectiva, Web semántica y Ludificación, para la captura, codificación y diseminación de buenas prácticas de agricultura VT38-UNLP10729 - Agroknowledge), se investiga en la realización de una wiki semántica para que la comunidad pueda organizar y contribuir en el conocimiento y divulgación de las BPA con foco en las prácticas del campo de tipo intensivas.

Este proyecto es dirigido por el Centro de Investigaciones LIFIA, de la Facultad de Informática (UNLP) y la participación de docentes investigadoras de la Facultad de Ciencias Agrarias y Forestales (UNLP). Se contó, además, con la colaboración de la de Dirección de Horticultura, fruticultura y floricultura del Ministerio de Agroindustria Provincia de Buenos Aires. Forma parte del trabajo realizado en el marco del Proyecto RUCAPS (Enhancing and Implementing Knowledge based ICT solutions within high Risk and Uncertain Conditions for Agriculture Production Systems, Horizon 2020, UE, Marie Curie Actions).

Esta wiki, realizada en base al framework de desarrollo MediaWiki (MediaWiki, s. f.), tiene por objetivo documentar, divulgar, y establecer un nexo para que la comunidad hortícola conozca y divulgue las BPA y al mismo tiempo pueda colaborar con la construcción de conocimiento sobre este conjunto de métodos. 


\section{Análisis de ludificación Wiki BPAi desde G.A.M.E.}

Con el objetivo de motivar a que una mayor cantidad de actores de la comunidad con el eje en la horticultura, participen y colaboren en la wiki de Buenas Prácticas Agrointensivas, BPAi, se diseñan algunas estrategias de ludificación.

Como primer recurso se analiza desde el marco conceptual genérico MDA los elementos de ludificación que mejor se adaptarían en un entorno colaborativo. Como segundo paso se analiza la propuesta de diseño conceptualmente, considerando la propuesta por etapas que propone G.A.M.E. el cual adquiere las siguientes características:

I. Para comenzar a diseñar un entorno ludificado, se evalúa el escenario de la comunidad en donde se propone abordar las BPAi, que, a los fines de este primer estudio, se focaliza en la zona del cinturón verde de La Plata. En esta zona se desarrollan entre sus producciones más importantes hortalizas (Garat et al., 2009). Las primeras fuentes de investigación surgen desde los aportes del equipo que integra el grupo de trabajo de Agroknowledge, entre los que se cuenta con representantes del ámbito académico, como así también representantes de trabajadores del sector. Como resultado de estas observaciones se considera que la comunidad de productores agrícolas de la región no está formada por usuarios frecuentes a este tipo de plataformas. Este análisis de carácter cualitativo, fue realizado como un primer acercamiento para potencialmente identificar el perfil de la comunidad BPAi.

II. A partir de identificar ciertos rasgos en la comunidad, se establece que resulta necesario potenciar en la misma el trabajo cooperativo y colaborativo, más que construir pequeñas individualidades. Esto se debe a que el cuerpo de conocimiento sobre lo que son las BPAi y cómo deben aplicarse, se encuentra aún en construcción, y resulta necesario motivar a que se logren construir colaborativamente, para así garantizar que puedan abarcar ampliamente las múltiples variables que tiene en cuanto campo de conocimiento.

III. Por lo tanto, en el momento de modelar, tomando en consideración el esquema de interacción de juego, se propone de la siguiente manera: El colaborador (jugador) realiza aportes en la wiki (acción) con el objetivo de generar mayor conocimiento sobre este campo (estado), lo que le devuelve una serie de indicios de que la wiki está creciendo (respuestas). Estos indicios dan cuenta de que los aportes que realizan los colaboradores en la wiki, tiene una consecuencia directa en el crecimiento de la comunidad. Por este motivo el elemento principal de ludificación que se implementa es el progreso, pero aplicado a la salud general de la wiki y no al progreso individual de cada usuario. El progreso generalmente se muestra en acciones de ludificación y en videojuegos como una barra de progreso, es decir una representación numérica o visual de lo avanzado en la acción/juego para lograr completar una misión (Buckley et al., 2019). En BPAi, este elemento se implementa desde dos ejes: A) un progreso en la salud de la página, es decir cada nueva página en la wiki cuando recién es creada comienza con un estado (semilla). Luego, este estado pasa al siguiente (primera floración) mientras sigue aumentando proporcionalmente al conte- 
nido y la información en la misma que es aportado, por los diferentes los colaboradores de la comunidad. Finalmente, se desarrolla un sistema de votación para que los usuarios puedan evaluar y validar en conjunto, si efectivamente esa página se corresponde con una BPAi, y así pasar al siguiente y último estado (maduración de la fruta), que evidencia que la página está correcta, completa y es una BPAi validada. B) el segundo eje a través del cual se diseña el elemento del progreso, es a través de la implementación de una interfaz robótica que muestra la salud total de la wiki. Es decir, el objetivo del diseño robótico es vincular sus estados y acciones al movimiento y crecimiento general de la wiki: a mayor cantidad de páginas creadas y validadas (en estado maduración del fruto) y mayor cantidad de usuarios activos que participan, más movimiento y acciones tendrá el robot.

IV. Luego de establecer el diseño y conceptualización mediante un marco de desarrollo, la implementación de estos parámetros se encuentra en etapa de prototipo. Para el prototipo se realiza una wiki BPAi espejo, en paralelo, en donde se actualizan e implementan únicamente los elementos de ludificación. Esta estrategia permite primero organizar funcionalmente y visualmente, cómo se incluirán los diversos elementos, pero sin riesgo de que el software de la wiki que se encuentra activa en etapa de carga de datos, pueda sufrir alguna pérdida de información por incompatibilidades con las interfaces de programación de aplicaciones, (application programming interface API). Este conjunto de aplicaciones, permite integrar en el software mediaWiki, propiedades o funcionalidades que no trae directamente incorporadas (Interfaz de programación de aplicaciones, 2019). Como se observa en la ilustración 3, los primeros testeos se centralizan en verificar que la API se incorpore correctamente a la interfaz de MediaWiki.

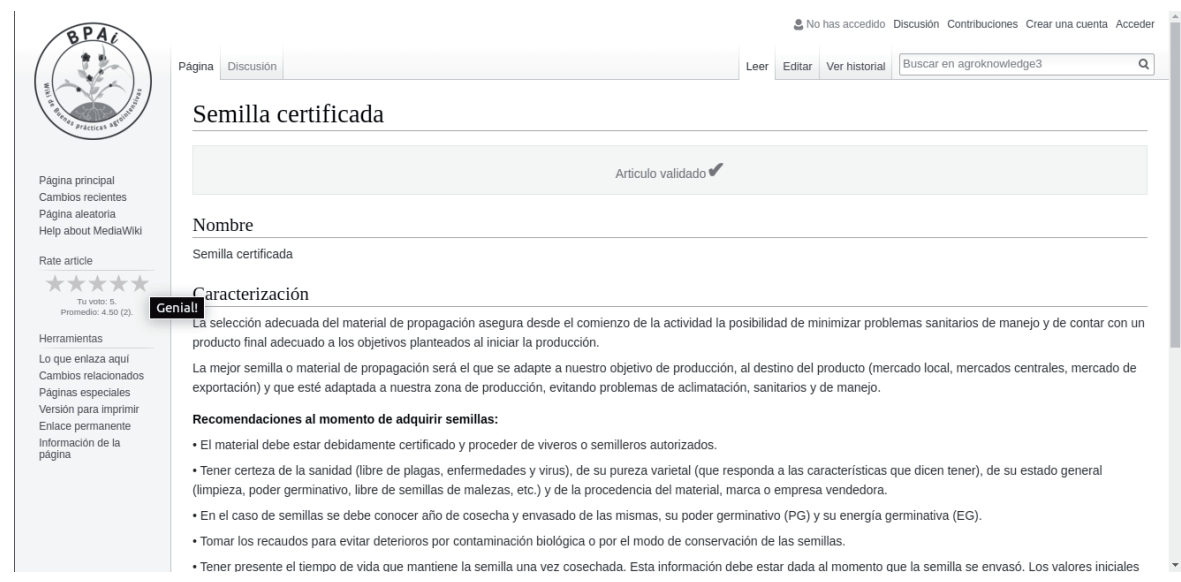

Ilustración 3: Captura de pantalla de prueba de página validada, sobre wiki espejo. 
Con estos primeros pasos, guiados desde G.A.M.E., se diseñan conceptualmente las mecánicas y las dinámicas que se incorporan a una Wiki semántica que busca incentivar el trabajo colaborativo. El objetivo es que, en términos de MDA, la estética en los usuarios funcione para profundizar la cooperatividad y el desafío.

\section{Diseño de una ludificación robótica}

El segundo eje entonces desde el cual se plantea la ludificación de BPAi es una instalación robótica. El diseño de esta interfaz tiene como objetivo vincular desde un parámetro físico, el progreso general de la wiki.

El diseño consta de un robot, cuya carcasa y estructura general se encuentra integrado a una planta. El robot vinculado a los datos que extrae de la wiki BPAi está provisto de sensores que pueden medir el estado de la planta que tiene incorporado y de luces de tipo LEDs. En su estructura formal remite a una forma redondeada, representando una abstracción de la planta de tomate. Así mismo el robot se desplaza sobre una superficie de tierra, la cual permite que pueda dejar surcos a medida que se mueva.

La selección de una interfaz robótica como complemento de la ludificación de la web, se presenta para promover una mayor personalización y empatía.

La empatía y la tecnología han estado vinculadas desde la primera herramienta creada por el hombre (Manney, 2008). Pero es en las tecnologías digitales que adquiere una nueva dimensión puesto que la opción a la acción genera además de una identificación, una proyección por parte del usuario dentro de la experiencia digital (Papale, 2014).

Siguiendo este eje, son dos los aspectos claves que se articulan al incorporar una interfaz robótica: por un lado, un eje relacionado a los videojuegos, en donde la posibilidad de abordar la narrativa a través de la acción, es decir, a través de las decisiones que el usuario puede optar y que modifican el desarrollo de la experiencia, promueve una mayor empatía generando una proyección de sí mismos dentro del escenario de juego. Por otro lado, un eje vinculado al arte robótico, que en cuanto artilugio o artefacto, al contar en su diseño con la capacidad de simular acciones que remiten a algo vivo, genera también una proyección de sí mismo por parte de los usuarios o espectadores al otorgarle al robot capacidades de resolución y autonomía que quizás no posee (Mondada \& Legon, 2001).

Por lo tanto, la figura del robot funciona como un avatar. Buckley et al. (2019) propone que en un espacio ludificado un avatar es una representación visual del jugador y define que el implementar esa figura en el diseño de juego sirve para personalizar la experiencia individual en una actividad ludificada, lo cual aumenta, por ende, el apego emocional a dicha actividad. Desde este sentido, el robot personaliza no a un individuo en particular, sino a la wiki en cuanto comunidad. Las decisiones que elige el robot para su desplazamiento son algoritmos que derivan de los cómputos que calcula según la actividad de los usuarios en un determinado lapso temporal. El objetivo de que la apariencia externa al robot se vincule directamente con una planta tiene una relación directa con el tipo de comunidad de práctica con la cual se relaciona, en este caso desde el área de los agricultores. 
Asimismo, acompañando su desplazamiento, los patrones lumínicos se manifiestan como códigos de comunicación. Cada acción que se realiza en la wiki modela un determinado patrón: Cada nuevo usuario ejecuta una figura de luces que se manifiesta como una línea que recorre todo el cuerpo del robot y asciende; cada nueva página determina los valores cromáticos; la edición en las páginas realiza una secuencia como efecto ola. La intensidad de las luces está dada por un promedio que calcula el robot desde la web de la wiki, en donde parametriza la actividad que tuvo en un breve lapso temporal. Si la wiki no tiene actividad, la luz irá disminuyendo en intensidad hasta apagarse.

Cabe destacar que la relación de la luz en el robot se basa en relaciones derivadas en el cultivo de plantas en contextos de interior. En estos trabajos se observa que el color de la luz tiene una incidencia sobre el crecimiento de las plantas (Massa et al., 2008). Por lo tanto, al momento de buscar un elemento para visualizar las acciones de los usuarios y continuar con el flujo del modelo de interacción del juego, se selecciona un elemento que tenga incidencia directa sobre la interfaz física, en este caso la planta que vive en el robot.

\section{Conclusiones y trabajo a futuro}

Para el presente artículo se abordó conceptualmente el diseño ludificado para una comunidad de construcción de conocimiento específica. Se considera este diseño desde un doble eje, el que se ejecuta directamente sobre la web en la wiki y un eje que se visualiza desde una interfaz robótica. A los fines de este artículo, no se profundiza en el corpus teórico y de investigación que acompaña el diseño robótico, el cual está en desarrollo y testeo. El objetivo principal es dar cuenta de la implementación de un marco de trabajo específicamente diseñado para las experiencias ludificadas colaborativas y que complementa el marco genérico MDA.

Como análisis reflexivo sobre la utilización de G.A.M.E. como marco de diseño, se desprende:

- G.A.M.E. resulta un marco conceptual amplio que, en los pasos que propone, hace énfasis en el análisis de la comunidad de usuarios. En este punto se contempla como trabajo futuro profundizar sistemáticamente desde un abordaje cualitativito en la investigación de la comunidad de practica vinculada a la BPA.

- G.A.M.E. también conforma un complemento en la utilización de un marco genérico como MDA. La estrategia de articular ambos marcos de referencia resulta en disponer más herramientas para abordar un diseño de juego que demanda características específicas y que en vez de observar un usuario en forma individual, trabaja con grupos de individuos. La secuencia que propone G.A.M.E. ofrece un espacio para el análisis y el modelado en función de las características que adquiere la comunidad como grupo. Asimismo, además de localizar estas características, el objetivo que se propone al implementar una ludificación, resulta fundamental para seleccionar las estéticas en términos de MDA que se busca como diseño final. 
Con respecto al objetivo de juego, en el diseño de la experiencia en BPAi se desprende fundamentalmente la interacción activa por parte de la comunidad en la wiki, como así también un desarrollo de conocimiento cooperativo, dado el impacto directo de las BPA con la práctica del agro a nivel mundial. Con la implementación del diseño ludificado, este objetivo se traduce para el usuario en proponerle:

a) Que logre el crecimiento de la Wiki, y de esta forma crecerá el conocimiento sobre las BPA. Esta escena se encuentra simbolizada en el juego a través de avatares de semillas de plantas de tomate. Cada nueva página una semilla, cada acción en las páginas avanza al siguiente estado de la planta (semilla, primera floración, maduración del fruto). Este objetivo se ve reflejado directamente en la interfaz de la web.

b) Que sostenga la vida del robot, por ende, de la planta que integra su estructura. Cuanto más movimiento hay en la wiki, la interfaz robótica tendrá más acciones, más estados, representados en movimiento y patrones lumínicos según la acción (nuevos usuarios, nuevas páginas, ediciones y aportes). De esta manera al crecer muchas plantas de frutos, en consecuencia, se lograr que el robot se desplace y viva. Este objetivo se ve reflejado en la interfaz física, el robot.

Como trabajo futuro se propone continuar con el paso IV de G.A.M.E. realizando testeos sistemáticos en diferentes grupos de usuarios específicos de la comunidad. El correcto registro y análisis de estos resultados, es el inicio de la etapa final, la de implementación sobre la web, para ser abordada finalmente en forma abierta y colaborativa. En cuanto a la interfaz física, en un siguiente análisis se propone abordar el detalle de su diseño, patrones de movimiento y marco conceptual, una vez finalizadas las primeras pruebas prototipo. Resta también profundizar en la vinculación de esta práctica robótica ludificada con el campo del bioarte, y la relación que puede establecerse con sostener la salud de la planta mediante las acciones que pueden elegir realizar los usuarios.

\section{Agradecimientos}

Este trabajo fue financiado parcialmente por la Secretaría de Políticas Universitarias, Programa Agregando Valor, proyecto Agroknowledge (Código 11059 entre 1/9/2018 al 31/10/2019), y realizado en el marco del Proyecto RUCAPS (Enhancing and Implementing Knowledge based ICT solutions within high Risk and Uncertain Conditions for Agriculture Production Systems, Horizon 2020, UE, Marie Curie Actions).

\section{Bibliografía}

Azouz, O. y Lefdaoui, Y. (2018). Gamification design frameworks: A systematic mapping study. 2018 6th International Conference on Multimedia Computing and Systems (ICMCS), 1-9. Recuperado de: https://doi.org/10.1109/icmcs.2018.8525900 
Brito, J., Vieira, V., \& Duran, A. (2015). Towards a Framework for Gamification Design on Crowdsourcing Systems: The G.A.M.E. Approach. 2015 12th International Conference on Information Technology - New Generations, 445-450. https://doi.org/10.1109/ ITNG.2015.78

Buckley, P., Noonan, S., Geary, C., Mackessy, T., \& Nagle, E. (2019). An Empirical Study of Gamification Frameworks. Journal of Organizational and End User Computing (JOEUC), 31(1), 22-38.

Buenas prácticas agrícolas (2019). Wikipedia. https://es.wikipedia.org/w/index. php?title=Buenas_pr\%C3\%A1cticas_agr\%C3\%ADcolas\&oldid $=121829980$

Crowdsourcing (2020). Wikipedia, la enciclopedia libre. Recuperado el 7 de Febrero de 2020 de https://es.wikipedia.org/wiki/Crowdsourcing

Cugelman, B. (2013). Gamification: What it is and why it matters to digital health behavior change developers. JMIR Serious Games, 1(1), e3-e3. PubMed. https://doi.org/10.2196/ games.3139

Deterding, S., Dixon, D., Khaled, R. y Nacke, L. (2011). From Game Design Elements to Gamefulness: Defining «Gamification». Proceedings of the 15th International Academic MindTrek Conference: Envisioning Future Media Environments. 9-15. https://doi. org/10.1145/2181037.2181040

Featured articles. (2020). Wikipedia. Recuperado el 7 de Febrero de 2020 de https:// en.wikipedia.org/w/index.php?title=Wikipedia:Featured_articles

Garat, J. J., Ahumada, A., Otero, J., Terminiello, L., Bello, G. y Ciampagna, M. L. (2009). Las hortalizas típicas locales en el cinturón verde de La Plata: Su localización, preservación y valorización. Horticultura Argentina, 28(66), 32-39.

Hunicke, R., LeBlanc, M., \& Zubek, R. (2004, July). MDA: A formal approach to game design and game research. Proceedings of the AAAI Workshop on Challenges in Game AI.

Interfaz de programación de aplicaciones (2019). Wikipedia. Recuperado el 7 de Febrero de 2020 de https://es.wikipedia.org/w/index.php?title=Interfaz_de_programaci\%C3\%B3n_ de_aplicaciones\&oldid $=122204387$

Landers, R. N., Auer, E. M., Collmus, A. B. y Armstrong, M. B. (2018). Gamification science, its history and future: Definitions and a research agenda. Simulation \& Gaming, 49(3), 315-337.

Lindsey, D. (2010). Evaluating quality control of Wikipedia's feature articles. First Monday, 15(4). Recuperado de https://doi.org/10.5210/fm.v15i4.2721

Manney, P. J. (2008). Empathy in the Time of Technology: How Storytelling is the Key to Empathy. Journal of Evolution \& Technology, 19(1).

Massa, G. D., Kim, H.-H., Wheeler, R. M. y Mitchell, C. A. (2008). Plant Productivity in Response to LED Lighting. HortScience, 43(7), 1951-1956. Recuperado de https://doi. org/10.21273/HORTSCI.43.7.1951

MediaWiki. (s. f.). Recuperado 25 de enero de 2020, de https://www.mediawiki.org/wiki/ MediaWiki

Mondada, F. y Legon, S. (2001). Interactions between Art and Mobile Robotic System Engineering. T. Gomi (Ed.) Evolutionary Robotics. From Intelligent Robotics to Artificial Life (pp. 121-137). Springer: Berlin. 
Mora, A., Riera, D., González, C., \& Arnedo-Moreno, J. (2017). Gamification: A systematic review of design frameworks. Journal of Computing in Higher Education, 29(3), 516-548. Recuperado de https://doi.org/10.1007/s12528-017-9150-4

Morschheuser, B., Hamari, J., \& Koivisto, J. (2016, January). Gamification in crowdsourcing: a review. In 2016 49th Hawaii International Conference on System Sciences (HICSS) (pp. 4375-4384). IEEE.

Morschheuser, B., Hamari, J., Koivisto, J. y Maedche, A. (2017). Gamified crowdsourcing: Conceptualization, literature review, and future agenda. International Journal of HumanComputer Studies, 106, 26-43.

Morschheuser, B., Hamari, J. y Maedche, A. (2019). Cooperation or competition - When do people contribute more? A field experiment on gamification of crowdsourcing. International Journal of Human-Computer Studies, 127, 7-24. Recuperado de https://doi. org/10.1016/j.ijhcs.2018.10.001

Narayan, S., Orlowitz, J., Morgan, J., Hill, B. M., \& Shaw, A. (2017, February). The Wikipedia Adventure: field evaluation of an interactive tutorial for new users. Proceedings of the 2017 ACM Conference on Computer Supported Cooperative Work and Social Computing (pp. 1785-1799).

Oceja, J., \& Sierra-Obregón, Á. (2018, October). Gamifiying Wikipedia?. In European Conference on Games Based Learning (pp. 504-XXII). Academic Conferences International Limited.

Papale, L. (2014, junio 22). Beyond Identification: Defining the Relationships between Player and Avatar. Journal of Games Criticism. Recuperado de: http://gamescriticism. org/articles/papale-1-2

Outreach Wiki. (s. f.). The Wikipedia Adventure. Recuperado 23 de enero de 2020. Recuperado de: https://outreach.wikimedia.org/wiki/The_Wikipedia_Adventure

Torres, D., Díaz, A., Cepeda, V., Correa, F. y Fernández, A. (2017). Nodos: Encyclopedia of the Performing Arts. Revista Colombiana de Computación, 18(2), 33-46. Recuperado de https://doi.org/10.29375/25392115.3216

Uchoa, A. P., Esteves, M. G. P. y Souza, J. M. de. (2013). Mix4Crowds - Toward a framework to design crowd collaboration with science. Proceedings of the 2013 IEEE 17th International Conference on Computer Supported Cooperative Work in Design (CSCWD), 61-66. Recuperado de https://doi.org/10.1109/CSCWD.2013.6580940

Wenger, E. (2020). Communities Of Practice: A Brief Introduction. STEP Leadership Workshop. University of Oregon. Recuperado de https://scholarsbank.uoregon.edu/ xmlui/handle/1794/11736

The Distributed Game. (s. f.). Wikidata. Recuperado 11 de abril de 2019. Recuperado de https://tools.wmflabs.org/wikidata-game/distributed/

Wikipedia. (2020) Wikipedia. Recuperado el 7 de Febrero de 2020. Recuperado de https:// es.wikipedia.org/w/index.php?title=Wikipedia

WikiCup (2020). Wikipedia. Recuperado de https://en.wikipedia.org/w/index. php?title=Wikipedia:WikiCup

Wöhner, T., \& Peters, R. (2009, October). Assessing the quality of Wikipedia articles with lifecycle based metrics. Proceedings of the 5th International Symposium on Wikis and Open Collaboration (pp. 1-10). 


\begin{abstract}
Currently, the game is analyzed as an important element to motivate the interaction of users in different types of environments. This analysis has resulted in promoting different strategies to adapt elements of games and video games in contexts that are not designed as such, but in which it seeks to stimulate the participation of users. One strategy when applying game elements is to consider a user experience-centric model.

However, to establish a game design in contexts where the collaborative aspect prevails, it is necessary to address specific strategies focused on your relationship with the community to which you belong. This article introduces the design of a gaded environment based on the G.A.M.E. conceptual framework for gathering, analysis, modeling, and execution, applied to a collaborative building community of knowledge of Good Horticultural Practices, complemented by a physical interface
\end{abstract}

Keywords: ludification - citizen science - collaborative work - Wikipedia - conceptual framework

Resumo: Atualmente, o jogo é analisado como um elemento importante para motivar a interação dos usuários em diferentes tipos de ambientes. Essa análise resultou na promoção de diferentes estratégias para adaptar elementos de jogos e videogames em contextos que não são desenhados como tal, mas nos quais busca estimular a participação dos usuários. Uma estratégia na aplicação de elementos de jogo é considerar um modelo centrado na experiência do usuário.

No entanto, para estabelecer um design de jogo em contextos onde o aspecto colaborativo prevalece, é necessário abordar estratégias específicas focadas em seu relacionamento com a comunidade à qual você pertence. Este artigo introduz o desenho de um ambiente gaded baseado no quadro conceitual G.A.M.E. para coleta, análise, modelagem e execução, (gathering, analysis, modeling and execution) aplicado a uma comunidade de construção colaborativa de conhecimento de Boas Práticas Horticulturais, complementado por uma interface física.

Resumo: ludificação - ciência cidadã - trabalho colaborativo - Wikipedia - estrutura conceitual

[Las traducciones de los abstracts fueron supervisadas por el autor de cada artículo] 
